\title{
Topic Set Size Design for Paired and Unpaired Data
}

\author{
Tetsuya Sakai \\ Waseda University, Tokyo, Japan \\ tetsuyasakai@acm.org
}

\begin{abstract}
Topic set size design is an approach to determining the sample sizes of an experiment (e.g., number of topics) based on a statistical requirement, namely a desired statistical power or a cap on the confidence interval (CI) width for the difference in means. Previous work considered paired data cases for a desired power of the $t$ test and for a cap on CI width, as well as unpaired data cases for a desired power of one-way ANOVA. In the present study, we consider unpaired (i.e., two-sample) cases for the $t$-test and for the CI width. Since one-way ANOVA with two groups is strictly equivalent to the two-sample $t$-test, we compare the outcomes of the topic set size design results based on these two approaches, and show that the one-way ANOVA-based approach actually returns tighter sample sizes than the two-sample $t$-test approach. Moreover, we compare the paired and unpaired cases for both $t$-test-based and CI-based topic set size design approaches. Because estimating the variance of the score differences for the paired data setting is problematic, we recommend the use of our unpaired-data versions of $t$-test-based and CI-based topic set size design tools, as they only require a variance estimate for individual scores and the appropriate sample sizes for unpaired data are also large enough for paired data.
\end{abstract}

\section{KEYWORDS}

confidence intervals; effect sizes; evaluation; sample sizes; statistical power; statistical significance; test collections

\section{ACM Reference Format:}

Tetsuya Sakai. 2018. Topic Set Size Design for Paired and Unpaired Data. In ICTIR '18: 2018 ACM SIGIR International Conference on the Theory of Information Retrieval, September 14-17, 2018, Tianjin, China. ACM, New York, NY, USA, 4 pages. https://doi.org/10.1145/3234944.3234971

\section{INTRODUCTION}

Sakai's Microsoft Excel tools for topic set size design [7] are simple implementations of sample size design techniques described in Nagata [5]. They aim to provide a straight answer to test collection builders' frequently asked question: "How many topics should I create in order to guarantee reliable experiments?" His first tool computes the required topic set size to ensure high statistical power for the paired $t$-test; his second tool does the same for one-way ANOVA with $m$ groups (e.g., systems); his third tool provides the required topic set size to ensure a small confidence interval (CI)

Permission to make digital or hard copies of all or part of this work for personal or classroom use is granted without fee provided that copies are not made or distributed for profit or commercial advantage and that copies bear this notice and the full citation on the first page. Copyrights for components of this work owned by others than the author(s) must be honored. Abstracting with credit is permitted. To copy otherwise, or republish, to post on servers or to redistribute to lists, requires prior specific permission and/or a fee. Request permissions from permissions@acm.org.

ICTIR '18, September 14-17, 2018, Tianjin, China

(c) 2018 Copyright held by the owner/author(s). Publication rights licensed to ACM ACM ISBN 978-1-4503-5656-5/18/09 . \$ \$15.00

https://doi.org/10.1145/3234944.3234971 width for the difference between any two systems for paired data. As the $t$-test and the CI-based tools are for paired data, they require a variance estimate $\hat{\sigma}_{d}^{2}$ of the difference between two systems in terms of a particular evaluation measure. Whereas, the ANOVAbased tool is for unpaired data, and requires a variance estimate $\hat{\sigma}^{2}$ of each system under the homoscedasticity assumption: that is, the scores of each system are assumed to obey possibly different normal distributions but with a common variance $\sigma^{2}$.

In the present study, we introduce two topic set size design tools for unpaired (i.e., two-sample) data, one based on a desired power of $t$-test and one based on a cap on the CI width of the differences in means. Sakai [7] considered the paired data case only as IR researchers often conduct paired data experiments using test collections, where different systems are evaluated using a common topic set. However, unpaired settings are also possible, for example, when User Group A rates System 1 and User Group B rates System 2, and we are interested in the appropriate group sizes. We then directly compare topic set size design based on the unpaired $t$-test with the existing one-way ANOVA-based approach, by leveraging the fact that one-way ANOVA with $m=2$ groups is strictly equivalent to the unpaired $t$-test. Based on our analysis, we recommend the use of the ANOVA-based tool with $m=2$ groups even when the researcher is interested in topic set size design for paired or unpaired $t$-tests, as it provides the tightest sample size estimates. Moreover, we compare the paired and unpaired cases for both $t$-test-based and CI-based topic set size design approaches. Because estimating the variance $\sigma_{d}^{2}$ of the score differences for the paired data setting is problematic, we recommend the use of our unpaired-data versions of $t$-test-based and CI-based topic set size design tools over the paired-data ones, as they only require a variance estimate $\sigma^{2}$ for individual scores, and the appropriate sample sizes for unpaired data are always large enough for paired data as well.

\section{PRIOR ART}

Until around early 1990s, IR researchers were on the whole reluctant to apply parametric significance tests such as $t$-tests and ANOVAs to IR evaluation, and preferred the nonparametric alternatives such as the sign test. For example, Van Rijsbergen remarked in 1979 [10] (p.136): "Parametric tests are inappropriate because we do not know the form of the underlying distribution." In the same year, in their discussion of designing "ideal" IR test collections, Gilbert and Sparck Jones [3] reported on a table that shows the required number of topics as a function of the number of relevant or retrieved documents per topic, also based on the sign test. They remarked: "Since there is some doubt about the feasibility of getting 1000 requests, or the convenience of such a large set for future experiments, we consider 500 requests." In 1993, Hull [4] advocated the use of parametric significance tests; perhaps from around that time, more and more IR researchers started to accept these tests. With the advent to computer-based, distribution-free tests, namely, 
the bootstrap [6] and the randomisation test [9], it is now easy for researchers to demonstrate the robustness of the classical parametric tests to distributional assumption violations. While statistical signifance testing is just a tool (See, for example, Carterette [1]), and harm can be done if used inappropriately (See, for example, Ziliak and McCloskey [13]), our view is that it can be a useful tool if researchers use it appropriately by considering and reporting statistical power, effect sizes, and confidence intervals.

With the exception of the TREC Million Query track which once had about 1,800 "sparsely-judged" topics to evaluate a given set of participating runs [2], the de facto standard in IR evaluation has been to use test collections that each contain a set of 50 topics, despite the aforementioned considerations for "ideal" test collections in the 1970s. Researchers who worried about this default topic set size have conducted topic set splitting experiments to empirically examine the reliability of such topic sets. For example, Voorhees [11] concluded that "Fifty-topic sets are clearly too small to have confidence in a conclusion when using a measure as unstable as $P(10)$."

Webber, Moffat, and Zobel [12] proposed an incremental method for constructing a topic set for a test collection where a topic (with relevance assessments) is added to the set one by one until the desired statistical power for the paired $t$-test is achieved. Whereas, Sakai's topic set size design approach [7] aims to provide simple answers to questions such as: "How many topics should I prepare if I want the paired $t$-test for any system pair with an effect size of 0.2 to achieve $80 \%$ statistical power while ensuring that the Type I error probability is no more than $5 \%$ ?" The present study builds on this approach.

\section{POWER-BASED TOPIC SET SIZE DESIGN}

This section first introduces a topic set size design tool based on the two-sample $t$-test. This enables us to directly compare one-way ANOVA-based $t$-test-based approaches, for one-way ANOVA with $m=2$ groups is exactly equivalent to the two-sample $t$-test. More specifically, it is known that if a random variable $t$ obeys a $t$ distribution with $\phi$ degrees of freedom, $t^{2}$ obeys an $F$ distribution with $(1, \phi)$ degrees of freedom. Moreover, when $m=2$, the $F$ statistic for one-way ANOVA is exactly the square of the $t$ statistic for the two-sided, two-sample $t$-test. Whereas, the power calculations for $t$-tests and ANOVA are done differently [5], so we should examine which approach gives us tighter sample size estimates. This is discussed in the latter half of this section. In addition, we also compare the results with the paired $t$-test-based approach.

\subsection{Topic Set Size Design based on the Two-Sided, Two-Sample $t$-test}

The model behind the two-sample $t$-test is as follows. The scores $x_{1 j}$ (e.g., nDCGs) of System 1 independently obey $N\left(\mu_{1}, \sigma^{2}\right)(j=$ $\left.1, \ldots, n_{1}\right)$; the scores $x_{2 j}$ of System 2 independently obey $N\left(\mu_{2}, \sigma^{2}\right)$ $\left(j=1, \ldots, n_{2}\right)$, where $n_{1}, n_{2}$ are the sample sizes (e.g., topic set sizes, user group sizes). The null hypothesis is $H_{0}: \mu_{1}=\mu_{2}$, and the alternative hypothesis is $H_{1}: \mu_{1} \neq \mu_{2}$ (a two-sided test). Let $\bar{x}_{1 \bullet}=\sum_{j=1}^{n_{1}} x_{1 j} / n_{1}, \quad \bar{x}_{2 \bullet}=\sum_{j=1}^{n_{2}} x_{2 j} / n_{2}$ and

$$
V_{p}=\frac{\sum_{j=1}^{n_{1}}\left(x_{1 j}-\bar{x}_{1 \bullet}\right)^{2}+\sum_{j=1}^{n_{2}}\left(x_{2 j}-\bar{x}_{2 \bullet}\right)^{2}}{\phi}, \quad \phi=n_{1}+n_{2}-2
$$

Given the above distributional assumptions, the following random variable obeys a noncentral $t$ distribution with $\phi$ degrees of freedom with a noncentrality parameter $\lambda_{t}[5]$ :

$$
t_{0}=\frac{\bar{x}_{1 \bullet}-\bar{x}_{2 \bullet}}{\sqrt{V_{p}\left(1 / n_{1}+1 / n_{2}\right)}},
$$

where

$$
\lambda_{t}=\sqrt{\frac{n_{1} n_{2}}{n_{1}+n_{2}}} \Delta_{t}, \quad \Delta_{t}=\frac{\mu_{1}-\mu_{2}}{\sigma} .
$$

Eq. 2 is the usual $t$-value we compute when conducting a twosample $t$-test. Under $H_{0}$, the noncentrality parameter $\lambda=0$ and therefore $t_{0}$ obeys the central $t$-distribution, a special case of the noncentral $t$ distribution. On the other hand, Under $H_{1}, \lambda \neq 0$ and $t_{0}$ obeys a (truly) noncentral $t$ distribution. We are interested in the latter situation, for statistical power is the ability to detect a difference when there in fact is a difference.

For any random variable $t^{\prime}$ that obeys the aforementioned noncentral $t$ distribution, $\operatorname{Pr}\left(t^{\prime} \leq w\right)$ for any $w$ can be approximated as follows [5], using a random variable $u$ that obeys a standard normal distribution:

$$
\operatorname{Pr}\left(u \leq \frac{w c^{*}-\lambda_{t}}{\sqrt{1+w^{2}\left(1-c^{* 2}\right)}}\right) \approx \operatorname{Pr}\left(u \leq \frac{w(1-1 / 4 \phi)-\lambda_{t}}{\sqrt{1+w^{2} / 2 \phi}}\right),
$$

where

$$
c^{*}=\frac{\sqrt{2} \Gamma((\phi+1) / 2)}{\sqrt{\phi} \Gamma(\phi / 2)},
$$

and $\Gamma(\bullet)$ denotes the gamma function [5]. Hence, the statistical power of a two-sided, two-sample $t$-test can be approximated as:

$$
\operatorname{Pr}\left(u \leq \frac{-w(1-1 / 4 \phi)-\lambda_{t}}{\sqrt{1+w^{2} / 2 \phi}}\right)+1-\operatorname{Pr}\left(u \leq \frac{w(1-1 / 4 \phi)-\lambda_{t}}{\sqrt{1+w^{2} / 2 \phi}}\right),
$$

where $w=t_{\text {inv }}(\phi ; \alpha)$, the two-sided $100 \alpha \% t$-value for $\phi$ degrees of freedom, since we make decisions based on a central $t$ distribution in significance testing, regardless of what $t_{0}$ actually obeys.

To discuss appropriate topic set sizes for the two-sample $t$-test, we consider a balanced design, $n=n_{1}=n_{2}$, so that:

$$
\phi=2 n-2, \quad \lambda=\sqrt{\frac{n}{2}} \Delta_{t}
$$

from Eqs. 1 and 3. Given $\alpha$ (Type I error probability), $\beta$ (Type II error probability, i.e., one minus statistical power), and the minimum detectable difference $\min D_{t}$ between two systems, a good initial sample size estimate can be obtained as [5]:

$$
n \approx 2\left(\frac{z_{i n v}(\alpha / 2)-z_{i n v}(1-\beta)}{m i n D_{t} / \sqrt{\hat{\sigma}^{2}}}\right)^{2}+\frac{z_{\text {inv }}(\alpha / 2)^{2}}{4},
$$

where $z_{\text {inv }}(P)$ denotes the upper $100 P \% z$-value and $\hat{\sigma}^{2}$ is an estimate of the aforementioned common variance for a particular evaluation measure. That is, we try to ensure $100(1-\beta) \%$ power whenever the true difference $\left(\mu_{1}-\mu_{2}\right)$ between any two systems is $\min D_{t}$ or larger. Given $\left(\alpha, \beta, \min D_{t}, \hat{\sigma}^{2}\right)$, we first compute Eq. 8 as an initial estimate, and check with Eq. 6 to see whether the desired power is actually achieved. If not, we increment the estimate by one, and repeat the process. Our Excel tool automates this process. 


\subsection{One-way ANOVA vs. $t$-tests}

One-way ANOVA-based topic set size design requires the following input: $\alpha$ and $\beta$ as before, the number of systems to be compared $(\mathrm{m})$, and the minimum detectable range $(\mathrm{min} D)$, and the variance estimate $\left(\hat{\sigma}^{2}\right)$ : we try to guarantee $100(1-\beta) \%$ power whenever the population means of the best and the worst among the $m$ systems differ by $\min D$. Clearly, when $m=2, \min D$ is equivalent to the aforementioned minimum detectable difference, $\min D_{t}$. Moreover, as was mentioned earlier, one-way ANOVA with $m=2$ groups (e.g., systems) is equivalent to the two-sample $t$-test: the only difference is that the $F$ statistic used in one-way ANOVA is a squared $t$ statistic. Hence, theoretically, the above two-sample $t$-test-based tool and Sakai's one-way ANOVA-based one (when $m$ is set to 2) should return an identical topic set size for the same setting of $\left(\alpha, \beta, \min D_{t}, \hat{\sigma}^{2}\right)$. In practice, however, the results slightly differ, as we shall demonstrate below, for the power calculations are done differently: while the $t$-test power calculation involves approximating a noncentral $t$ distribution as discussed above, ANOVA-based power calculation involves approximating a noncentral $F$ distribution [7].

We also compare topic set size results based on the paired and two-sample $t$-tests. Given accurate variance estimates, the paired $t$-test should require a small sample size than the two-sample $t$ test under the same statistical requirement. However, while we can easily obtain an unbiased estimate of the variance $\hat{\sigma}^{2}$ for both two-sample and one-way ANOVA tests by computing the residual variance given a topic-by-run score matrix [7], the paired $t$-testbased topic set size design requires a variance estimate $\hat{\sigma}_{d}^{2}$ for the score differences. The paired $t$-test assumes $N\left(\mu_{1}, \sigma_{1}^{2}\right)$ and $N\left(\mu_{2}, \sigma_{2}^{2}\right)$ for the scores of the two systems and therefore $N\left(\mu_{1}-\mu_{2}, \sigma_{1}^{2}+\sigma_{2}^{2}\right)$ for the score differences; hence Sakai [7] recommends letting $\hat{\sigma}_{d}^{2}=2 \hat{\sigma}^{2}$ without heeding the covariance, to consciously overestimate $\sigma_{d}^{2}$ and err on the side of oversampling. Under this setting, how will the results based on paired and two-sample $t$-tests differ?

Following Sakai [7], we address the above questions using residual variances from past TREC data for four different evaluation measures: AP (Average Precision), Q (Q-measure), nDCG (normalised Discounted Cumulative Gain), and nERR (normalised Expected Reciprocal Rank): from the topic-by-run score matrices of the TREC 2003 and 2004 robust tracks (with document cutoff 1000), we obtained the following pooled variances $\hat{\sigma}^{2}$ for the four measures: 0.047, 0.047, 0.046, 0.115; similarly, from the TREC 2011 and 2012 web adhoc tasks (with document cutoff 10), we obtained 0.082, 0.037, $0.044,0.086$. We then let $\hat{\sigma}_{d}^{2}=\hat{\sigma}^{2}$ for the paired $t$-test-based topic set size design.

Table 1 summarises our power-based topic set size design results. The paired $t$-test-based and ANOVA-based results were obtained using Sakai's Excel tools [7]; the two-sample $t$-test-based results were obtained using our tool. It can be observed that the ANOVAbased tool returns the tightest estimates despite the fact that the two-sample $t$-test and one-way ANOVA with $m=2$ groups are strictly equivalent. The differences arise from the respective power calculation methods. Moreover, the paired and two-sample $t$-test results are almost identical: the former does not return smaller topic set sizes, due to our overestimation of $\sigma_{d}^{2}$. These results suggest that we can use the ANOVA-based tool with $m=2$ even if we are interested in the power of paired or two-sample $t$-tests.
Table 1: Power-based topic set size design results: paired $t$ test/two-sample $t$-test/one-way $\operatorname{ANOVA}(m=2)$.

\begin{tabular}{|c|c|c|c|c|}
\hline $\min _{t}$ & AP & Q & nDCG & nERR \\
\hline \multicolumn{5}{|c|}{ TREC $2003+2004$ robust (cutoff=1000) } \\
\hline 0.05 & $298 / 297 / 289$ & $298 / 297 / 289$ & $291 / 290 / 283$ & $725 / 724 / 705$ \\
0.10 & $76 / 75 / 73$ & $76 / 75 / 73$ & $75 / 74 / 71$ & $183 / 182 / 177$ \\
0.15 & $35 / 34 / 33$ & $35 / 34 / 33$ & $35 / 34 / 32$ & $83 / 82 / 79$ \\
0.20 & $21 / 20 / 19$ & $21 / 20 / 19$ & $21 / 20 / 19$ & $48 / 47 / 45$ \\
0.25 & $14 / 13 / 12$ & $14 / 13 / 12$ & $14 / 13 / 12$ & $31 / 30 / 29$ \\
\hline \multicolumn{5}{|c|}{ TREC 2011+2012 web adhoc (cutoff=10) } \\
\hline 0.05 & $517 / 516 / 503$ & $235 / 234 / 227$ & $279 / 278 / 270$ & $542 / 541 / 527$ \\
0.10 & $131 / 130 / 126$ & $61 / 60 / 58$ & $72 / 71 / 68$ & $137 / 136 / 133$ \\
0.15 & $60 / 59 / 57$ & $28 / 27 / 26$ & $33 / 32 / 31$ & $62 / 61 / 59$ \\
0.20 & $35 / 34 / 32$ & $17 / 16 / 15$ & $20 / 19 / 18$ & $36 / 35 / 34$ \\
0.25 & $23 / 22 / 21$ & $12 / 11 / 10$ & $14 / 13 / 12$ & $24 / 23 / 22$ \\
\hline
\end{tabular}

\section{CI-BASED TOPIC SET SIZE DESIGN}

This section first introduces a topic set size design method based on a cap on the CI width for the difference between two systems when the data are unpaired, under the homoscedasticity assumption. Then a simple approximation is introduced to both paired and unpaired CI-based topic set size design tools, in order to make the tools capable of handling large topic set sizes. Finally, the CI-based topic set size results based on paired and unpaired settings are compared.

\subsection{Topic Set Size Design based on the CI Width for Unpaired Data}

In CI-based topic set size design, we try to ensure that the expected CI width for the difference between any system pair is no larger than $\delta$. As before, we try to find the right sample size $n$ under a balanced condition: $n=n_{1}=n_{2}, \quad \phi=2 n-2$. In this case, the above condition can be expressed as:

$$
2 t_{i n v}(\phi ; \alpha) E\left(\sqrt{V_{p}}\right) \sqrt{\frac{1}{n_{1}}+\frac{1}{n_{2}}}=2 t_{i n v}(\phi ; \alpha) c^{*} \sigma \sqrt{\frac{2}{n}} \leq \delta,
$$

where $c^{*}$ is given by Eq. 5 and $\sigma$ is the common standard deviation ${ }^{1}$.

Although Sakai [7] used Eqs. 5 and 9 to consider the appropriate CI-based topic set size for paired data, Eqs. 5 relies on the Gamma function, which is computationally highly expensive $e^{2}$. Hence we propose to utilise the following approximation:

$$
c^{*} \approx 1-1 / 4 \phi \text {. }
$$

In fact, this approximation has already been used for $t$-test-based topic set size design in Eq. 4, when we discussed replacing a random variable that obeys a noncentral $t$ distribution with one that obeys $N\left(0,1^{2}\right)$. Figure 1 compares $c^{*}$ as defined in Eq. 5 and its approximation as defined in Eq. 10. It can be observed that the approximation is highly accurate except when $\phi$ is extremely small. Hence, we use the above approximation for both paired-data and unpaired data cases in CI-based topic set size design.

Given the above approximation, Eq. 9 can be rewritten as:

$$
\frac{t_{i n v}(\phi ; \alpha)(1-1 / 4 \phi)}{\sqrt{n}} \leq \frac{\delta}{2 \sqrt{2} \sigma} \approx \frac{\delta}{2 \sqrt{2 \hat{\sigma}^{2}}}, \quad \phi=2 n-2 .
$$

${ }^{1}$ Note that $E\left(\sqrt{V_{p}}\right)=c^{*} \sigma$ while $E\left(V_{p}\right)=\sigma^{2}$. That is, $V_{p}$ is an unbiased estimator of $\sigma^{2}$, but $\sqrt{V_{p}}$ is not an unbiased estimator of $\sigma$.

${ }^{2}$ GAMMA (172) is greater than $10^{307}$ and cannot be computed by Excel. 


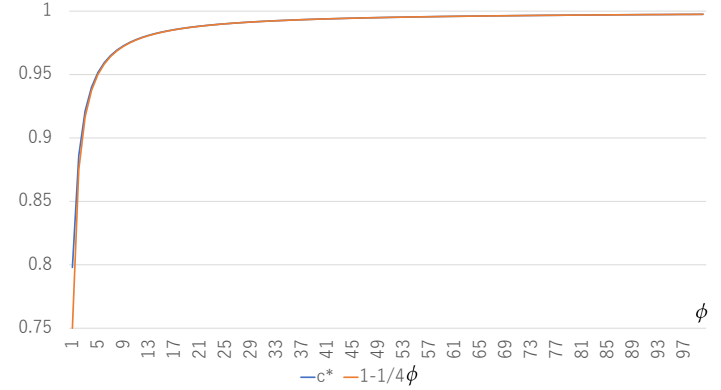

Figure 1: Accuracy of $c^{*} \approx 1-1 / 4 \phi$.

Table 2: CI-based topic set size design results: paired/unpaired data.

\begin{tabular}{|c|c|c|c|c|}
\hline$\delta$ & AP & Q & nDCG & nERR \\
\hline \multicolumn{5}{|c|}{ TREC $2003+2004$ robust (cutoff=1000) } \\
\hline 0.10 & $147 / 146$ & $147 / 146$ & $144 / 143$ & $356 / 355$ \\
0.20 & $39 / 38$ & $39 / 38$ & $38 / 37$ & $91 / 90$ \\
0.30 & $19 / 18$ & $19 / 18$ & $18 / 17$ & $42 / 41$ \\
0.40 & $12 / 11$ & $12 / 11$ & $11 / 10$ & $25 / 24$ \\
0.50 & $8 / 7$ & $8 / 7$ & $8 / 7$ & $17 / 16$ \\
\hline \multicolumn{5}{|c|}{ TREC $2011+2012$ web adhoc (cutoff $=10$ ) } \\
\hline 0.10 & $254 / 253$ & $116 / 115$ & $138 / 137$ & $267 / 266$ \\
0.20 & $65 / 64$ & $31 / 30$ & $36 / 35$ & $69 / 68$ \\
0.30 & $30 / 29$ & $15 / 14$ & $18 / 17$ & $32 / 31$ \\
0.40 & $18 / 17$ & $10 / 9$ & $11 / 10$ & $19 / 18$ \\
0.50 & $13 / 12$ & $7 / 6$ & $8 / 7$ & $13 / 12$ \\
\hline
\end{tabular}

The initial topic set size estimate, which is an optimistic estimate that uses a standard normal distribution $\left(\sigma^{2}\right.$ known) instead of a $t$-distribution ( $\sigma^{2}$ unknown), is given by [5]:

$$
n \approx \frac{8 z_{\text {inv }}(\alpha / 2)^{2} \hat{\sigma}^{2}}{\delta^{2}} .
$$

Then we check if this $n$ actually satisfies Eq. 11; if not, we increment $n$ and repeat the above. Our Excel tool automates this process.

For the paired data case, the following corresponds to Eq. 11:

$$
\frac{t_{i n v}(\phi ; \alpha)(1-1 / 4 \phi)}{\sqrt{n}} \leq \frac{\delta}{2 \sigma_{d}}, \quad \phi=n-1 .
$$

Note that, if we let $\sigma_{d}^{2}=2 \sigma^{2}$ as before, Eq. 13 becomes identical to Eq. 11 except for the degrees of freedom. As for the initial topic set size estimate, it is given by [7]:

$$
n \approx \frac{4 z_{i n v}(\alpha / 2)^{2} \hat{\sigma}_{d}^{2}}{\delta^{2}} .
$$

which again is identical to Eq. 12 if we let $\hat{\sigma}_{d}^{2}=2 \hat{\sigma}^{2}$. For the paired data case, we have verified that our new CI-based tool returns the same result as that of Sakai [7], and that it can return large topic set sizes where Sakai's cannot.

\subsection{Paired vs. Unpaired Data in CI-based Topic Set Size Design}

Table 2 compares the CI-based topic set size design results for paired and unpaired data settings, using the aforementioned variance estimates from TREC data. It can be observed that our unpaired data tool returns topic set sizes that are exactly one topic smaller than the corresponding paired data cases, even though theoretically paired data require smaller sample sizes than unpaired data. Again, this is due to our overestimation of $\sigma_{d}^{2}$.

\section{CONCLUSIONS AND LIMITATIONS}

We discussed the unpaired data cases for the $t$-test-based and CIbased topic set size design approaches. We directly compared the two-sample $t$-test approach and the one-way ANOVA-based approach (with $m=2$ ), and showed that the latter returns tighter sample sizes despite the fact that the two tests are strictly equivalent for $m=2$. Moreover, we showed that, if the variance $\sigma_{d}^{2}$ of the score difference between two systems is estimated as $\hat{\sigma}_{d}^{2}=2 \hat{\sigma}^{2}$, the paired and unpaired results are basically the same for both $t$-testbased and CI-based topic set size design. This strongly suggests that we are indeed overestimating $\sigma_{d}^{2}$ 's. We recommend the use of our unpaired versions of the $t$-test-based and CI-based tools over the paired versions because: (a) The unpaired versions give slightly tighter estimates while avoiding the use of $\hat{\sigma}_{d}^{2}$; and (b) Topic set sizes that are large enough for unpaired experiments always suffice for paired experiments as well. Finally, our new CI-based tools avoids computing the gamma function and thus can handle large sample sizes, unlike Sakai's earlier tool [7]. Our topic set design tools are available from: http://www.f.waseda.jp/tetsuya/tools.html; an extended discussion of this work will be given in a forthcoming book [8]. Limitations of the present study are as follows. Firstly, to estimate the variance of the score differences, we did not go beyond $\hat{\sigma}_{d}^{2}=2 \hat{\sigma}^{2}$. This should not stop researchers from trying to obtain more accurate estimates of $\sigma_{d}^{2}$ somehow and thereby obtain tigher sample size estimates for paired data settings. Secondly, topic set size design does not consider multiple comparison procedures [1], as statistical power is difficult to define in these settings where there are multiple null hypotheses.

\section{REFERENCES}

[1] Ben Carterette. 2012. Multiple testing in statistical analysis of systems-based information retrieval experiments. ACM TOIS 30, 1 (2012).

[2] Ben Carterette, Virgil Pavlu, Evangelos Kanoulas, Javed A. Aslam, and James Allan. 2008. Evaluation over Thousands of Queries. In Proceedings of ACM SIGIR 2008. 651-658.

[3] H. Gilbert and K. Sparck Jones. 1979. Statistical Bases of Relevance Assessment for the 'ideal' Information Retrieval Test Collection. Technical Report. Computer Laboratory, University of Cambridge, British Library Research and Development Report No. 5481.

[4] David Hull. 1993. Using Statistical Testing in the Evaluation of Retrieval Experiments. In Proceedings of ACM SIGIR '93. 329-338.

[5] Yasushi Nagata. 2003. How to Design the Sample Size (in fapanese). Asakura Shoten.

[6] Tetsuya Sakai. 2006. Evaluating Evaluation Metrics based on the Bootstrap. In Proceedings of ACM SIGIR 2006. 525-532.

[7] Tetsuya Sakai. 2016. Topic Set Size Design. Information Retrieval 19, 3 (2016), 256-283.

[8] Tetsuya Sakai. 2018. Laboratory Experiments in Information Retrieval: Sample Sizes, Effect Sizes, and Statistical Power. Springer.

[9] Mark D. Smucker, James Allan, and Ben Carterette. 2007. A Comparison of Statistical Significance Tests for Information Retrieval Evaluation. In Proceedings of ACM CIKM 2007. 623-632.

[10] C. J. van Rijsbergen. 1979. Information Retrieval. Butterworths, Chapter 7.

[11] Ellen M. Voorhees. 2009. Topic Set Size Redux. In Proceedings of ACM SIGIR 2009. 806-807.

[12] William Webber, Alistair Moffat, and Justin Zobel. 2008. Statistica Power in Retrieval Experimentation. In Proceedings of ACM CIKM 2008. 571-580.

[13] Stephen T. Ziliak and Deirdre N. McCloskey. 2008. The Cult of Statistical Significance: How the Standard Error Costs Us fobs, fustice, and Lives. The University of Michigan Press. 